\title{
On the time gating of SPADs in a synchronized time- gated SPAD array in Raman spectroscopy
}

\author{
Ilkka Nissinen, Jan Nissinen and Juha Kostamovaara \\ Circuits and Systems Research Unit \\ University of Oulu \\ Oulu, Finland \\ first name.last name@oulu.fi
}

\begin{abstract}
The effect of the timing of the biasing of a single photon avalanche diode (SPAD) on the accuracy of a time-gated SPAD array has been studied in this work. The measurement was realized in a time-gated Raman spectrometer utilizing a 16x256 CMOS SPAD array with on-chip time gating electronics. SPADs have to be biased in to the Geiger-mode just before arriving Raman scattered photons from the sample to achieve synchronous measurements with a pulsed laser, and to minimize the dark count noise. The practical realization is, however, not straight-forward due to the strict timing requirements $(\sim 50 \mathrm{ps})$. With the shown biasing arrangement high timing accuracy and low noise can be achieved without sacrificing the fill factor of the detector array.
\end{abstract}

Keywords - time interval measurement; timing error; time resolving $S P A D$

\section{INTRODUCTION}

Large single-photon avalanche diode arrays (more than hundreds of SPADs) have been developed for various applications, for example for fluorescence life time imaging, time-of-flight laser rangefinding, laser induced breakdown spectroscopy and for Raman spectroscopy $[1,2,3,4]$. Raman spectroscopy is one of the most recent applications where CMOS technology has been utilized to integrate the SPAD array with the time gating and the time interval measurement electronics on a single chip.

Raman spectroscopy is traditionally based on a CW laser with narrow linewidth which is used for sample excitation. Raman scattered photons (wavelength shifted from the excitation wavelength) are collected with a spectrograph and a position sensitive CCD. One of the main problems in Raman spectroscopy is the strong fluorescence background which in many measurement cases masks the much weaker Raman signal. However, the Raman scattering photons and fluorescence photons have different time responses. If a short laser pulse instead of a CW excitation is used, the Raman photons are scattered instantly with the excitation laser pulse but fluorescence photons have an exponentially decaying lifetime. Most of the fluorescence photons can be excluded from the result if photons are recorded within a short time gate corresponding with the laser pulse width, especially if the laser pulse width is much shorter than the fluorescence lifetime $[5,6]$.

As mentioned above, CMOS technologies can be used to fabricate SPAD arrays and additionally, all the electronics can be integrated in the same die which makes it possible to design quite large arrays with time gating electronics for Raman spectroscopy $[7,4]$.

This work was supported by the Academy of Finland (Centre of Excellence in Laser Scanning Research, contract no. 272196, and contract nos. 282405 and 292609)
Block and timing diagrams with three different biasing plans of a Raman spectrometer based on a pulsed laser and a timegated SPAD array are shown in Fig. 1 a) and b), respectively. To maximize the Raman signal collection, a synchronizing signal from the laser (Trigger in Fig. 1) is used to bias all the SPADs below the grating into the Geiger mode (in that mode SPADs can detect individual photons) before Raman photons arrive from the sample as is illustrated in Fig 1. b) with all the bias plans. If the bias plan 1 is used a lot of dark count induced noise is collected before the Raman photon collection window leading the poor signal-to-noise ratio. On the other hand, if bias plan 2 is used the noise level can be decreased but a very large switching transistor is needed to bias all the SPADs rapidly. This leads to a decreased fill factor in the SPAD array consisting normally several parallel elements in a single spectral point. The reason for this is the fact that the parasitic capacitance of the biased node has to be minimized to make biasing faster and therefore the biasing transistors have to be placed within the $\mathrm{SPAD}$ cells. The bias plan 3 is based on a much smaller bias transistor so that a higher fill factor can be achieved with fast enough biasing. This biasing plan also allows SPADs to detect photons during the biasing pulse, which will be explained in more detail below.

In this work, the effect of the timing of the bias plan 3 used in a $16 \times 256$ SPAD array $(0.35 \mu \mathrm{m}$ HVCMOS) on the performance of Raman spectroscopy was investigated in details. The effect of the timing of biasing was measured by using two samples: a fluorescence free sample, titanium oxide $\left(\mathrm{TiO}_{2}\right)$ and olive oil having fluorescence lifetime of approximately 2 ns.

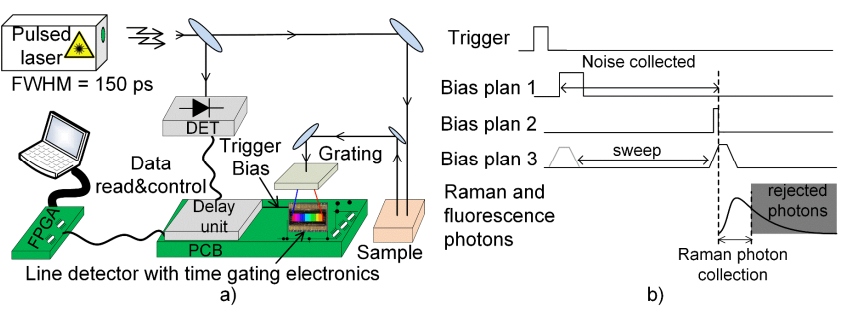

Fig. 1. a) Block diagram of the pulsed laser-based Raman spectrometer and b) timing diagram of different biasing signal possibilities.

\section{ACTIVE BIASING IN TIME-GATED RAMAN SPECTROMETER}

The line detector of time-gated Raman spectroscopy device consists of 16×256 SPADs which are actively biased and quenched during measurements to achieve synchronous measurements with the laser excitation. Fig. 2 a) and b) show schematic and timing diagrams of a single SPAD element with 
a biasing and quenching circuitry, respectively. A single SPAD is based on structure presented in [1]. Before a laser pulse the SPAD is kept quenched by connecting the anode node to a supply voltage of $3.3 \mathrm{~V}$ through the switch M1. When a trigger signal arrives from a laser, the quenching is stopped and after that the rising edge of $V_{\text {Bias }}$ starts to discharge the anode node to the ground through switch M2. The SPAD enters to the Geiger mode immediately with discharging if the high voltage of the cathode $\left(\mathrm{V}_{\mathrm{HV}}\right)$ is connected to the breakdown voltage of the $\mathrm{SPAD}+3.3 \mathrm{~V}$. Eventually, the SPAD has an excess bias of 3.3 $\mathrm{V}$ when the anode is discharged to the ground. The key issue here is that the transistor M2 is sized so that the resistance value of it (in triode region) is large enough to give a large enough signal to trig the buffer at the anode node even when it is closed (ON state) if the photon is detected by the SPAD during the biasing. Photons are collected by enabling the photon collection window by using D flip-flops as shown in Fig. 2 a) and b). The photon collection window is set to collect photons during the Raman scattering. A detected photon within the photon collection window is indicated by state 01 at the output of the flip-flops.
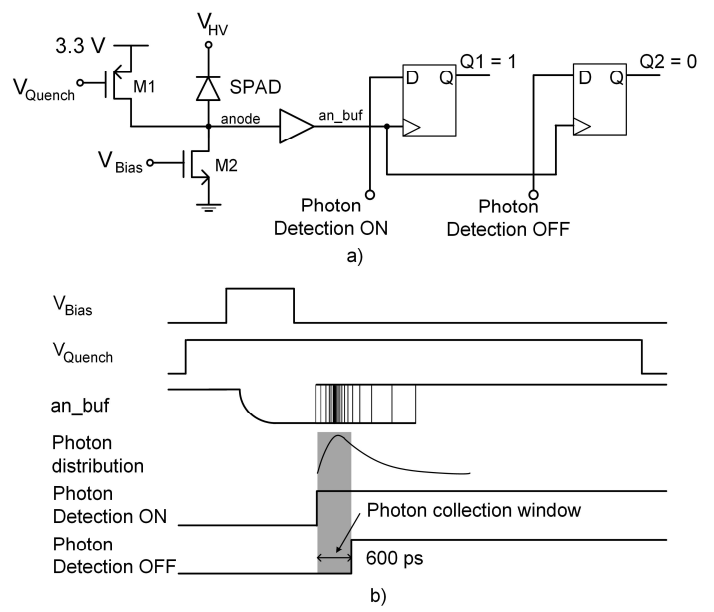

Fig. 2. Schematic and timing diagrams of the active quenching and biasing circuit.

\section{MEASUREMENT RESULTS}

Measurements were carried out with the system shown in Fig. 1 a) by using a laser having a pulse width and energy of 150 ps (FWHM) and $0.2 \mu \mathrm{J}$, respectively. The width of the biasing pulse was set to $3 \mathrm{~ns}$ and kept constant during all the measurements after the effect of the position of the falling edge of that pulse on the photon collection was tested. These tests showed that the effect of the position of the falling edge of the biasing pulse with the respect to a collection window was negligible compared to that of the rising edge of biasing pulse. This was expected because the falling edge of $V_{\text {bias }}$ is just opening the switch M2 after the anode node is discharged to ground and when it is closed the resistance value of it is enough large to cause the triggering of a buffer at the anode node which was planned by using the bias plan 3. The width of the Raman photon collection window was set to be approximately $600 \mathrm{ps}$. In that case, most of the Raman photons can be collected at every 256 spectral points during the window in spite of the window width variation and the timing skew of the window along a spectral axis which are caused by the mismatches of timing signals inside the chip [7].

An adjustable delay unit (Delay unit in Fig. 1 a)) was used to delay the trigger signal so that the timing of biasing of all the SPADs in a line detector can be swept as shown in Fig. 1 b) sweep. The sweep was started $7.5 \mathrm{~ns}$ before the center of the photon collection window and it was continued until the rising edge of the bias pulse reaches Raman photon collection window. The Raman spectra of titanium oxide and olive oil were recorded during sweeps so that the effect of the timing point of the bias pulse with relation to the Raman photon collection window on the performance of Raman spectroscopy could be evaluated.

\section{A. Normalized measurements with a fluorescent sample}

A fluorescent sample based on olive oil was measured to achieve a normalized photon collection intensity as a function of a spectral point i.e. pixel with the different time positions of the biasing pulse. Fig. 3 shows the normalized spectra of olive oil (without Raman peaks because of normalization) as a function of the position of the rising edge of a biasing pulse. As can be seen, the intensity of spectra are quite similar until the rising edge of a biasing pulse starts to be near the collection window edge at $5.5 \mathrm{~ns}$. After that the intensity is decreasing because of the finite fall time of the anode node. In other words, SPADs are not biased to the maximum excess bias voltage when photons start to occur within collection window. The intensity reaches zero when the rising edge passes the whole collection window whose center point was adjusted to be at $7.5 \mathrm{~ns}$ (the center of Raman photons too) resulting to the start and stop time of the collection window of $7.2 \mathrm{~ns}$ and $7.8 \mathrm{~ns}$, respectively. The fall time of anode node can be calculated be approximately $7.2 \mathrm{~ns}-$ $5.5 \mathrm{~ns}=1.7 \mathrm{~ns}$.

\section{B. Raman spectrum measurements}

The effect of a biasing pulse on the Raman spectrum was studied by using two samples, a fluorescent free sample, titanium oxide $\left(\mathrm{TiO}_{2}\right)$ and a high fluorescent sample, olive oil. In the measurements of $\mathrm{TiO}_{2}$ the load pulse was swept from 0 to $7.5 \mathrm{~ns}$ with a time step of 250 ps towards a photon collection window. The Raman spectra of $\mathrm{TiO}_{2}$ at different positions of the rising edge of a biasing pulse are shown in Fig. 4 a) (showing only pixels 0 - 100 to clarify Fig. 4 a)). Raman spectra marked by grey and black colors are derived using results with the rising edges of a biasing pulse from 0 to $5.5 \mathrm{~ns}$ (with $1 \mathrm{~ns}$ step) and from $6.5 \mathrm{~ns}$ to $7.5 \mathrm{~ns}$ (with $0.25 \mathrm{~ns}$ step), respectively.

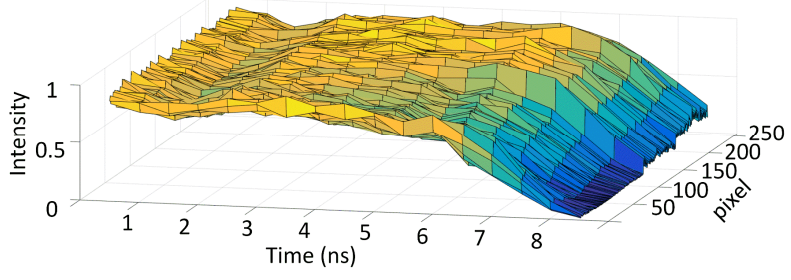

Fig. 3. Normalized spectra of olive oil.

Ideally, with very fast rising times all spectra should look similar with different time positions, until the rising edge of a biasing pulse passes the photon collection window. As can be seen in Fig. 4 a) the intensities of Raman peaks are quite similar when the time positions of the rising edge is between 0 and 5.5 
ns (grey curves), but intensities start to decrease rapidly after the SPAD cannot be biased properly because of finite rise time (black curves). The Raman peaks of $\mathrm{TiO}_{2}$ can be still distinguished because the dark count rate of the time-gated SPAD is much smaller than the Raman signal of the fluorescence free sample.

The same measurement was made using a highly fluorescent sample, olive oil. The Raman spectra of the olive oil with the different time positions of the rising edge of a biasing pulse can be seen in Fig. 4 b). Now, the Raman peaks start to vanish below the fluorescence when the rising edge of a biasing pulse arrives $1.5 \mathrm{~ns}$ before the center of the collection window (black curves), because the fluorescence noise level is much higher than the dark count noise level of SPADs. The Signal-to-fluorescence noise ratio of Raman spectrum as a function of the time position of the rising edge at the pixel 185 is also shown in small figure in Fig. 4 b). When fluorescence noise is dominating the SNR can be simply approximated by dividing the photon counts of Raman amplitude by the square root of the photon counts of fluorescence level next to Raman peak resulting in the SNR of $\sim 5$ at the pixel 185 when the rising edge is arriving at $7.5 \mathrm{~ns}$ (102/sqrt(390), 300000 laser pulses shot).
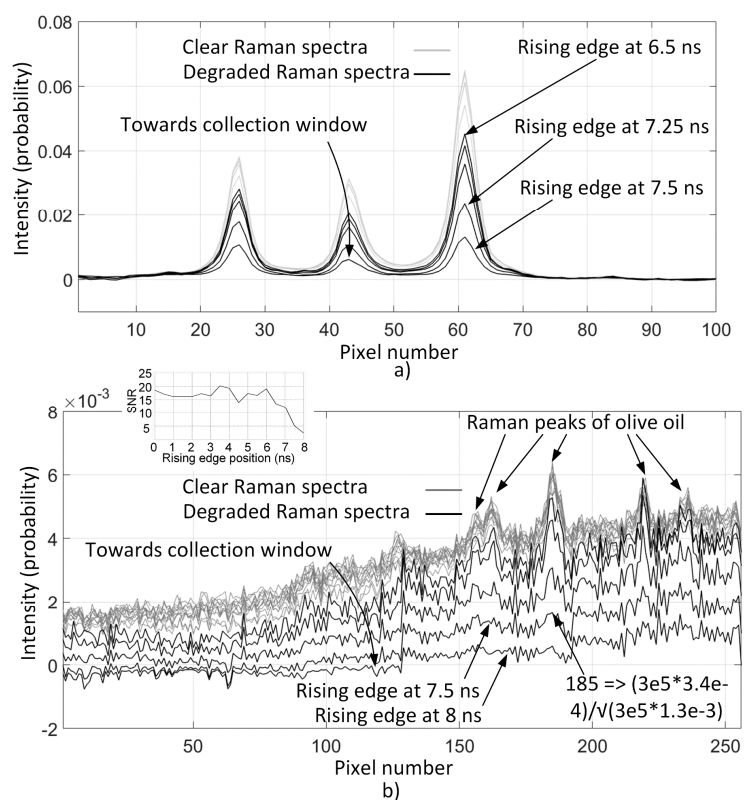

Fig. 4. Raman spectra of a) $\mathrm{TiO}_{2}$ and b) olive oil with different time positions of a biasing pulse.

\section{Time domain measurements}

The timing moment of the SPAD is shifted by the approaching biasing pulse and the finite rise time of the anode node of the SPAD. The shift of this timing of the triggering of arriving photons from the sample were measured by using six photon collection windows to cover the whole time period of $600 \mathrm{ps}$ and thus the individual window size was adjusted to be 100 ps. The photon distributions were collected by using those six collection windows at the one spectral point which has high Raman intensity of $\mathrm{TiO}_{2}$. Fig. 5 shows the time drift of the photon distribution as a function of the approaching biasing pulse (time sweep in Fig. 5). As can be seen the peak intensity is moved later when the bias pulse is approaching the collection window which was expected because the SPAD wasn't fully biased when the bias pulse is approaching the collection window slowing down the triggering.

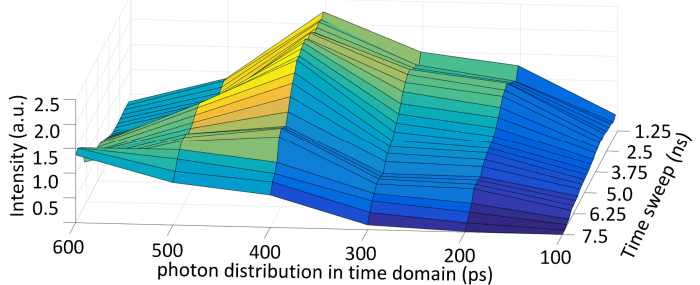

Fig. 5. Time domain photon distribution as a functon of the time position of the rising edge of a bias pulse.

\section{CONCLUSIONS}

It was shown that high-speed gating of a SPAD detector can be realized with a biasing pulse whose length is longer than the expected time window resolution by properly selecting the dimensions of the switching transistor. In this way the dark count induced noise can be minimized and fill factor maximized. The effect of the time position of the rising edge of this biasing pulse of the SPADs with relation to the photon collection window on the performance of the time-gated Raman spectroscopy was also studied. Measurements showed that the triggering moment of SPADs is started to delay by the rising edge of the biasing pulse when it arrives approximately $1.5 \mathrm{~ns}$ before the collection window in which case SPADs cannot be fully biased to the maximum excess bias before the collection window. However, the significant decrease of the SNR of the Raman spectrum of a high fluorescent sample cannot be seen until the rising edge of the biasing pulse arrives $1 \mathrm{~ns}$ before the center of a collection window of 600 ps.

\section{REFERENCES}

[1] D. Mosconi, D. Stoppa, L. Pancheri, L. Gonzo and A. Simoni, "CMOS single-photon avalanche diode array for time-resolved fluorescence detection", in Proc. IEEE European Solid-State Circuits Conference (ESSCIRC'06), pp. 564-567, Montreux, Switzerland, 2006.

[2] D. Stoppa et al. "A CMOS 3-D imager based on single photon avalanche diode," IEEE Transactions on Circuits and Systems I: Regular Papers, vol. 54, no. 1, pp. 4-12, Jan. 2007

[3] I. Nissinen et al., "A sub-ns time-gated CMOS single photon avalanche diode detector for Raman spectroscopy", in Proc. IEEE ESSDERC, pp. 375-378, 2011.

[4] Y. Maruyama, J. Blacksberg and E. Charbon, "A 1024x8, 700-ps timegated SPAD line sensor for planetary surface exploration with laser Raman spectroscopy and LIBS," IEEE Journal of Solid-State Circuits, vol. 49, no. 1, pp. 179-189, Jan. 2014.

[5] R.P. Van Duyne, D.L. Jeanmaire, and D.F. Shriver, "Mode-locked laser Raman spectroscopy-A new technique for the rejection of interfering background luminescence signals," Analytical Chemistry, vol. 46, no. 2, pp. 213-222, Feb. 1974.

[6] P. Matousek et al., "Fluorescence suppression in resonance Raman spectroscopy using a high-performance picosecond Kerr gate," Journal of Raman Spectroscopy,vol. 32, no. 12,pp. 983-988, Dec. 2001.

[7] I. Nissinen et al. ," A 2x(4)x128 multi-time-gated SPAD line detector for pulsed Raman spectroscopy", IEEE Journal of Sensors, vol. 15, no. 3, pp. 1358-1365, 2015. 